\title{
Modelamiento matemático de la cinética de secado de espagueti enriquecido con pulpa de zapallo deshidratada (Cucurbita moschata)
}

\section{Mathematical modeling of spaghetti drying kinetics enriched with dehydrated squash pulp (Cucurbita moschata)}

Natali López-Mejía ${ }^{1 *}$; Margarita María Andrade-Mahecha²; Hugo Alexander Martínez-Correa ${ }^{3}$

1'Ingeniera Agroindustrial, Magister en Ingeniería Agroindustrial, Estudiante Auxiliar de Investigación, Facultad de Ingeniería y Administración. Universidad Nacional de Colombia, Palmira, Colombia, e-mail: nalopezme@unal.edu.co, (DD https://orcid.org/0000-0001-9136-5205

${ }^{2}$ Ingeniera Agroindustrial, Doctora en Ingeniería de Alimentos, Profesora asociada de la Facultad de Ingeniería y Administración. Universidad Nacional de Colombia, Palmira, Colombia, e-mail: mmandradem@unal.edu.co, D https://orcid.org/0000-0002-6329-8022

${ }^{3}$ Ingeniero Químico, Doctor en Ingeniería de Alimentos, Profesor asociado de la Facultad de Ingeniería y Administración. Universidad Nacional de Colombia, Palmira, Colombia, e-mail: hamartinezco@unal.edu.co, (D) https://orcid.org/0000-0002-9617-0033

*autor de correspondencia: nalopezme@unal.edu.co

Cómo citar: López-Mejía, N.; Andrade-Mahecha, M.M.; Martínez-Correa, H.A. 2019. Modelamiento matemático de la cinética de secado de espagueti enriquecido con pulpa de zapallo deshidratada (Cucurbita moschata). Rev. U.D.C.A Act. \& Div. Cient. 22 (1):e1151. https://doi.org/10.31910/rudca.v22.n1.2019.1151

Artículo de acceso abierto publicado por Revista U.D.C.A Actualidad \& Divulgación Científica bajo una licencia Creative Commons CC BY-NC 4.0

Recibido: Junio 18 de 2018

Aceptado: Enero 22 de 2019

\section{RESUMEN}

A pesar que diversos estudios se han llevado a cabo sobre modelamiento matemático de las cinéticas de secado de espagueti, no se han desarrollado para espaguetis con sustitución de sémola de trigo Triticum durum por pulpa de zapallo deshidratada (PZD), por lo tanto, el objetivo de esta investigación fue modelar las cinéticas de secado de espagueti sustituido parcialmente con PZD (5 y 10g/100g harina), evaluar el efecto de la sustitución y la temperatura (50 y $60^{\circ} \mathrm{C}$ ), sobre el tiempo de secado (contenido de humedad final = $0,13 \mathrm{~g} / \mathrm{g}$ b.s.), la difusividad efectiva ( $\mathrm{D}_{\mathrm{e}}$ ) y algunas características que definen la calidad del producto (contenido de humedad, calidad de cocción y contenido de carotenoides totales). Para ello, se emplearon modelos matemáticos reportados en la literatura, como también la segunda ley de Fick, para un cilindro infinito. Los resultados mostraron que los modelos Henderson \& Pabis y el Logarítmico presentaron mayor ajuste $\left(\mathrm{R}^{2} \geq 0,90\right)$ a las cinéticas de secado experimentales; por el contrario, el modelo de Lewis presentó el menor ajuste. Se obtuvieron tiempos de secado de 5,00-4,10h, para las cinéticas realizadas a $50^{\circ} \mathrm{C}$ y tiempos de secado de $3,40-2,80 \mathrm{~h}$ (aproximadamente), para las cinéticas a $60^{\circ} \mathrm{C}$. De varió desde 1,50 hasta $2,50 \times 10^{-7} \mathrm{~cm}^{2} \mathrm{~s}^{-1}$, la cual, aumentó con la temperatura. En definitiva, la calidad del espagueti se afectó negativamente con el aumento de PZD y positivamente con el aumento de la temperatura de secado.

Palabras clave: modelos matemáticos, transferencia de masa; difusividad; sustitución; Cucurbita; Triticum durum. CAB Thesaurus. 


\section{ABSTRACT}

Although several studies have been carried out on mathematical modeling of the spaghetti drying kinetics, they have not been developed for spaghetti with an incorporation of Triticum durum wheat semolina by dehydrated squash pulp (DSP). Therefore, the objective of this investigation was to model the kinetics of spaghetti drying partially substituted with DSP (5 and $10 \mathrm{~g} / 100 \mathrm{~g}$ flour), to evaluate the effect of substitution and temperature $\left(50\right.$ and $\left.60^{\circ} \mathrm{C}\right)$, on the time of drying (final moisture content $=0.13 \mathrm{~g} / \mathrm{g} \mathrm{d} . \mathrm{b}$.), the effective diffusivity $\left(D_{e}\right)$ and some characteristics that define the quality of the product (moisture content, cooking quality and total carotenoid content). For this, mathematical models reported in the literature were used, as well as the second law of Fick for an infinite cylinder. The results showed that the Henderson \& Pabis and the Logarithmic models presented greater adjustment $\left(\mathrm{R}^{2} \geq 0.90\right)$ to the experimental drying kinetics. On the contrary, the Lewis model presented the smallest adjustment. Drying times of 5.00-4.10h were obtained for the kinetics carried out at $50^{\circ} \mathrm{C}$ and drying times of $3.40-2.80 \mathrm{~h}$ (approximately) for the kinetics at $60^{\circ} \mathrm{C}$. It varied from 1.50 to $2.50 \times 10^{-7} \mathrm{~cm}^{2} \mathrm{~s}^{-1}$, which increased with temperature. Finally, the spaghetti quality was negatively affected with the increase in PZD and positively with the increase in the drying temperature.

Keywords: mathematical models, mass transfer, diffusivity, substitution, Cucurbita, Triticum durum. CAB Thesaurus.

\section{INTRODUCCIÓN}

Las pastas alimenticias son consideradas un alimento de consumo masivo carente de micronutrientes importantes, como betacaroteno, con bajo contenido de fibra dietaria y bajo valor biológico de su proteína, al no tener aminoácidos esenciales, como la Lisina (Astaíza et al. 2010). La pulpa de zapallo es un material biológico de alto valor nutricional, ya que contiene minerales importantes para el desarrollo humano, como el fósforo y el zinc, betacaroteno (precursor de vitamina A) (Bergantin et al. 2018), compuestos fenólicos y alto contenido de fibra dietaria, que le proporciona actividad hipoglicemiante (Lu et al. 2019) e hipolipemiante (Aljahani \& Cheikhousman, 2017). Es por ello, que es una materia prima idónea para enriquecer una matriz alimentaria como el espagueti.

Algunos estudios han reportado el uso de la pulpa de zapallo en la formulación de pastas alimenticias (Padalino et al. 2016; Mirhosseini et al. 2015; Minarovičová et al. 2017); sin embargo, estos han sido en pastas frescas y no se ha evaluado el efecto de las condiciones de secado sobre las propiedades de esta, así como tampoco se han elaborado modelamientos matemáticos que describan las cinéticas de secado, siendo esta etapa tan importante.

Es sabido que el secado es un proceso regido por la transferencia de calor y de masa del alimento con el aire que lo rodea y consiste en remover la humedad libre contenida en el producto, a través de la evaporación del agua que, generalmente, se da mediante convección forzada (Ertekin \& Yaldiz, 2004). El secado, se lleva a cabo con el principal propósito de hacer el producto más estable durante el almacenamiento, prolongando así su vida útil. Otras ventajas del secado es que permite disminuir el peso del producto y, de esta forma, facilitar su transporte, reduciendo el espacio requerido para el almacenamiento (Vega \& Fito, 2005). Cuando se registra el contenido de humedad del producto en el transcurso del secado, se logra obtener la curva de secado, característica llamada curva experimental.

La curva característica de secado, se suele dividir en tres períodos: período de precalentamiento, velocidad de secado constante y velocidad de secado decreciente; sin embargo, los dos primeros son usualmente muy cortos, comparados con todo el período de secado en la producción de pasta seca, por lo tanto, los modelos reportados en la literatura están enfocados al período de velocidad de secado decreciente. Estos estudios contemplan modelos teóricos, semiteóricos y empíricos, para describir la transferencia de agua y la cinética del proceso de secado. La mayoría de los modelos teóricos utilizados en la ciencia de los alimentos se basan en las leyes de difusión de Fick y sus ecuaciones derivadas resueltas por Crank (1975), para tres formas geométricas: placa plana, cilindro y esfera. Adicional a ello, se encuentran ecuaciones empíricas o semiempíricas, para describir curvas de secado, como los son la de Newton (Westerman et al. 1973), Page (Agrawal \& Singh, 1977), Page modificada (Ozdemir \& Devres, 1999), Henderson \& Pabis (Chhinnan, 1984), Logarítmico (Yagcioglu et al. 1999), Dos Términos (Henderson, 1974) y Dos Términos Exponenciales (Sharaf-Eldeen et al. 1980), entre otros.

Generalmente, el secado de pastas alimenticias inicia con un contenido de humedad del producto del 31\% en base seca (b.s.) y finaliza alrededor del 13\% (b.s.); permite la formación de la estructura, lo que aumenta la consistencia, debido a la red proteica que genera el gluten junto con el almidón. A nivel industrial, se emplea aire caliente $\left(40-105^{\circ} \mathrm{C}\right)$, con humedades relativas, entre 40 y 85\% (De Temmerman et al. 2007). A pesar de que diferentes estudios se han llevado a cabo sobre modelamiento matemático de las cinéticas de secado de espagueti, pocos se han desarrollado para espaguetis con sustitución parcial o total de la sémola de trigo (T. durum), por otro tipo de harina. En la literatura, se encuentra el estudio llevado a cabo por Larrosa et al. (2016) para espagueti libre de gluten, elaborado con almidón de maíz, harina de maíz, huevo en polvo, entre otros; estos autores evaluaron cuatro condiciones de secado, combinando dos temperaturas $\left(30\right.$ y $\left.50^{\circ} \mathrm{C}\right)$ y dos humedades relativas (40 y $80 \%$ ). Los autores obtuvieron tiempos de secado entre 3,16-9,33h.

Modelar las cinéticas de secado de espagueti sustituido parcialmente con PZD (5 y 10g/100g harina), evaluar el efecto de la sustitución y la temperatura $\left(50\right.$ y $\left.60^{\circ} \mathrm{C}\right)$ sobre el tiempo de secado, la difusividad efectiva $\left(\mathrm{D}_{\mathrm{e}}\right)$ y algunas características que definen la calidad del producto, fueron los objetivos de este estudio. 


\section{MATERIALES Y MÉTODOS}

Material. El material experimental utilizado fue zapallo (Cucurbita moschata) de la variedad Bolo verde, obtenido de un mercado local en Candelaria (Valle del Cauca, Colombia) (CAVASA, plaza mayorista de mercado). El material fue procesado en la planta piloto BioValle (Ginebra, Valle del Cauca). Para la selección de los frutos, se determinaron características fisicoquímicas, tales como color $\left(L^{*}=58,42 \pm 2,96 ; a^{*}=19,16 \pm 2,55\right.$ y b*= 27,27 $\left.\pm 3,65\right)$, materia seca $(8,99 \pm 0,002 \mathrm{~g} / 100 \mathrm{~g}),{ }^{\circ}$ Brix $(4,23 \pm 0,42), \mathrm{pH}(6,84$ $\pm 0,23)$, carotenoides totales $(35,47 \mu \mathrm{g} / \mathrm{g})$, siguiendo los métodos AOAC (2003). Los frutos, se lavaron para retirar material extraño y, seguidamente, se desinfectaron con solución de hipoclorito (100ppm). Posteriormente, cada fruto se fraccionó en 8 partes similares, se retiró la semilla y se escaldó $\left(60^{\circ} \mathrm{C} / 10\right.$ minutos $)$ (Orrego et al. 2016). Se dejaron enfriar hasta temperatura de 25 $\pm 1^{\circ} \mathrm{C}$ y se separó la cáscara de la pulpa. La pulpa fue procesada en julianas $(7 \mathrm{~mm} \times 5 \mathrm{~mm} \times 50 \mathrm{~mm})$, para ser sometidas a secado, siguiendo el método reportado por Potosí-Calvache et al. (2017), con algunas modificaciones $\left(55^{\circ} \mathrm{C} / 3 \mathrm{~ms}^{-1} / 24 \mathrm{~h}\right)$. Para esto, se empleó un horno convectivo (DIDACONTROL, Colombia, 2016) y cada bandeja $\left(4 \mathrm{~m}^{2}\right)$, previamente desinfectada, fue cargada con $2,70 \mathrm{~kg}$ de pulpa fresca. Una vez seco (humedad final $=13,08 \pm 0,07 \mathrm{~g} / 100 \mathrm{~g}$ b.s.), el material se sometió a molienda, en un molino de cuchillas (DIDACONTROL, Colombia, 2016), se tamizó, se empacó en recipientes de vidrio color ámbar y se almacenó a $8 \pm 1{ }^{\circ} \mathrm{C}$.

Caracterización de la materia prima. La pulpa de zapallo deshidratada y la sémola de trigo fueron caracterizadas. Se cuantificaron por triplicado propiedades, contenido de humedad, proteína, fibra dietaria, cenizas, grasa (AOAC, 2003) y carotenoides totales (Ordoñez-Santos et al. 2014).

Elaboración del espagueti. El espagueti fue elaborado, según De Pilli et al. (2009), con algunas modificaciones. La formulación base (sémola de trigo T. durum: $35 \mathrm{~g} / 100 \mathrm{~g}$ mezcla y agua destilada: $65 \mathrm{~g} / 100 \mathrm{~g}$ mezcla) fue modificada, con la incorporación de pulpa de zapallo deshidratada (5 y $10 \mathrm{~g} / 100 \mathrm{~g}$ de harina), denominados respectivamente PZD-5 y PZD-10. Cada componente, se pesó en una balanza de precisión (Mettler Toledo, PB1502, Suiza) y se mezcló manualmente, hasta lograr una apariencia uniforme. Después, se adicionó agua destilada, se amasó por $15 \mathrm{~min}$, hasta obtener una masa elástica y se dejó reposar por $1 \mathrm{~h}$ a $8,00 \pm 0,50^{\circ} \mathrm{C}$ (Mabe, In-genius, México), previamente recubierta con papel aluminio. Seguidamente, la masa se laminó en una máquina manual (Imperia, Italia, 2015) y se moldeó en forma de cilindros tipo espagueti $($ diámetro $=2,00 \pm 0,50 \mathrm{~mm}$ y largo $=100 \pm 5 \mathrm{~mm})$.

Curvas experimentales de secado. El secado de cada formulación de espagueti, se realizó en una cámara climática (MEMMERT, ICH 260L, Alemania). En este proceso, todas las formulaciones de espagueti fueron evaluadas en dos condiciones ( $\mathrm{T} / \mathrm{HR}$ ), con base en lo reportado por Dexter et al. (1981), con algunas modificaciones: - Condición 1: $50^{\circ} \mathrm{C} / 50 \% \mathrm{HR}(0,33 \mathrm{~h})$ (etapa I), 50 ${ }^{\circ} \mathrm{C} / 70 \% \mathrm{HR}$ (6h) (etapa II) y $40^{\circ} \mathrm{C} / 50 \% \mathrm{HR}(12 \mathrm{~h}$ ) (etapa III).
- Condición 2: $50^{\circ} \mathrm{C} / 50 \% \mathrm{HR}(0,33 \mathrm{~h})$ (etapa I), $60^{\circ} \mathrm{C} / 70 \% \mathrm{HR}$ (6 h) (etapa II) y $40^{\circ} \mathrm{C} / 50 \% \mathrm{HR}(12 \mathrm{~h})$ (etapa III).

Para cada condición anteriormente mencionada, se emplearon rampas de secado, llevadas a cabo en tres etapas:

Etapa I, denominada pre-secado: En esta etapa, el contenido de humedad del espagueti se redujo hasta un $20 \%$, aproximadamente, y se realizó a baja temperatura, con el fin de evitar agrietamiento de la superficie y por un corto tiempo, para impedir el desarrollo de hongos.

Etapa II, denominada secado: Generalmente, se realiza a una temperatura más alta que la del pre-secado y tiene una duración aproximada de 5 a $6 \mathrm{~h}$, hasta llegar a un contenido de humedad del $13 \%$ (b.s.).

Etapa III, denominada estabilización: Consiste en mantener el espagueti a unas condiciones de baja temperatura y humedad relativa, con el fin de estabilizarlo, para posteriormente empacarlo y almacenarlo.

Cada condición de secado se realizó por triplicado, con una carga inicial de $300 \mathrm{~g}$ (espagueti húmedo), que se dispuso en un soporte fabricado en acero inoxidable.

Determinación del contenido de humedad. El contenido de humedad fue medido en el espagueti por triplicado, desde el tiempo 0 hasta el tiempo $6(\mathrm{~h})$, por el método de estufa, a $105^{\circ} \mathrm{C}$, durante $24 \mathrm{~h}$ (AOAC, 2003). Para ello, las muestras fueron rotuladas y guardadas por 1 día en cajas Petri plásticas, selladas y congeladas a $-20^{\circ} \mathrm{C}$.

Determinación de la humedad de equilibrio y razón de humedad. La humedad de equilibrio ( $\mathrm{Me})$, se halló determinando el contenido de humedad del espagueti correspondiente a cada experimento después de ser sometido a secado por 18h. Dicho valor fue utilizado para calcular la razón de humedad, la cual, se estimó mediante la ecuación 1, reportada por Geankoplis (1998).

$$
M R=\frac{M_{t}-M_{e}}{M_{0}-M_{e}}
$$

ecuación 1

Donde, $\mathrm{M}_{\mathrm{t}}$ es la humedad en el tiempo t, $\mathrm{M}_{\mathrm{o}}$ la humedad inicial y Me la humedad de equilibrio (g/g) (b.s.).

Modelamiento matemático de las curvas de secado y determinación del coeficiente de difusividad. El proceso de secado presenta, generalmente, un breve período de velocidad constante $y$, posteriormente, se produce un período de velocidad decreciente, donde el factor que controla es la difusión.

El modelado del período de velocidad constante, se realizó mediante un ajuste lineal de tipo $\mathrm{MR}=\mathrm{k}^{*} \mathrm{t}+\mathrm{b}$, donde $\mathrm{MR}$ es la humedad adimensional, $\mathrm{k}$ la pendiente $\mathrm{y} \mathrm{b}$ la intersección con el eje " $\mathrm{y}$ ". El grado de ajuste de los datos experimentales fue evaluado con la tendencia de la ecuación, obtenida mediante el coeficiente de determinación $\left(\mathrm{R}^{2}\right)$. 
El modelado del período de velocidad decreciente, se llevó a cabo mediante la segunda Ley de Fick, ecuación 2, para estado no estacionario en un cilindro infinito

$$
\frac{\partial \mathrm{C}}{\partial \mathrm{t}}=\mathrm{D}_{\mathrm{e}} \frac{\partial^{2} \mathrm{C}}{\partial^{2} \mathrm{r}^{2}}
$$

ecuación 2

Donde, $\mathrm{C}$ es el contenido de humedad de la muestra, De es el coeficiente de difusión $\left(\mathrm{m}^{2} \mathrm{~s}^{-1}\right)$, t es el tiempo en horas (h) y $r$ es la distancia desde el centro (radio) (m). La teoría matemática de difusión formulada por Fick y desarrollada por Crank (1975), presentada como la difusión, a través de un cilindro, como se evidencia en la ecuación 3.

$$
\frac{\partial \mathrm{C}}{\partial \mathrm{t}}=\frac{1}{\mathrm{r}} \frac{\partial}{\partial \mathrm{r}}\left(\mathrm{rD} \frac{\partial \mathrm{C}}{\partial \mathrm{r}}\right)
$$

ecuación 3

Se asumió el coeficiente de difusión constante en términos de las coordenadas cilíndricas $r, \theta$, z. En el caso de un cilindro circular largo, donde la difusión de agua se produce sobre toda la superficie, la ecuación 4 de difusión se reduce a:

$$
\frac{\partial \mathrm{C}}{\partial \mathrm{t}}=\frac{1}{\mathrm{r}} \frac{\partial}{\partial \mathrm{r}}\left(\mathrm{rD} \frac{\partial \mathrm{C}}{\partial \mathrm{r}}\right)
$$

ecuación 4
Se consideró que el contenido de humedad dentro de la pasta era uniforme a $\mathrm{t}=0$

$$
\mathrm{M}=\mathrm{M}_{0} \quad \text { para } 0<\mathrm{r}<\mathrm{r}_{\mathrm{c}}
$$

Donde, Mo representa el contenido de humedad de la pasta cuando inicia el secado y $\mathrm{r}_{\mathrm{c}}$ es el radio del cilindro que representa al espagueti.

La solución analítica para el secado de muestras cilíndricas desarrollada por Crank (1975), se muestra en la ecuación 5.

$$
\frac{\mathrm{M}_{\mathrm{t}}-\mathrm{M}_{\mathrm{e}}}{\mathrm{M}_{0}-\mathrm{M}_{\mathrm{e}}}=4 \sum_{\mathrm{n}=1}^{\infty} \frac{1}{\mathrm{~b}^{2}} \mathrm{e}^{\left[-\frac{\mathrm{b}^{2} \mathrm{Det}_{\mathrm{e}}}{\mathrm{r}^{2}}\right]}
$$

ecuación 5

Donde, De es el coeficiente de difusión efectiva $\left(\mathrm{m}^{2} \mathrm{~s}^{-1}\right), \mathrm{M}_{\mathrm{t}, 0}$ y e es el contenido de humedad en el tiempo t, $0 \mathrm{~h}$ y de equilibrio; t es el tiempo (h) y r es el radio (m).

La cinética de secado, también se ajustó mediante diferentes modelos matemáticos, mostrados en la tabla 1. Se seleccionó el modelo que mejor representó la curva de secado experimental y mediante los valores de MR obtenidos, se calculó el coeficiente de difusividad efectiva $\left(\mathrm{D}_{\mathrm{e}}\right)$, empleando la ecuación 5.

Tabla 1. Ecuaciones de las curvas de secado.

\begin{tabular}{|l|l|r|}
\hline $\begin{array}{c}\text { No. } \\
\text { Modelo }\end{array}$ & \multicolumn{1}{|c|}{ Modelo } & Ecuación del Modelo \\
\hline 01 & Lewis & MR $=\exp (-\mathrm{kt})$ \\
\hline 02 & Page & MR $=\exp \left(-\mathrm{kt} \mathrm{n}^{\mathrm{n}}\right)$ \\
\hline 03 & Henderson \& Pabis & $\mathrm{MR}=\mathrm{a} \exp (-\mathrm{kt})$ \\
\hline 04 & Logarítmico & $\mathrm{MR}=\mathrm{a} \exp (-\mathrm{kt})+\mathrm{b}$ \\
\hline 05 & Dos Términos & $\mathrm{MR}=\mathrm{a} \exp (-\mathrm{kot})+\mathrm{b} \exp (-\mathrm{k} 1 \mathrm{t})$ \\
\hline 06 & Dos Términos Exponencial & MR $=\mathrm{a} \exp (-\mathrm{kt})+(1 \mathrm{a}) \exp (-\mathrm{kat})$ \\
\hline
\end{tabular}

MR: Razón de Humedad; t: tiempo de secado (h); k: constante de secado; a, b, c y n son coeficientes de ajuste.

Caracterización de la calidad del espagueti. La calidad del espagueti seco fue medida en cuanto a contenido de humedad (AOAC, 2003), calidad de cocción (tiempo de cocción, ganancia de peso y sólidos perdidos en el agua de cocción) (AACCI 66-50, 2000) y contenido de carotenoides totales (Ordoñez-Santos et al. 2014), por triplicado.

Diseño experimental y análisis estadístico. Se evaluó el efecto de PZD (factor 1) y de la temperatura de secado (factor 2), en algunas propiedades que definen la calidad del espagueti (calidad de cocción y contenido de carotenoides totales). Para ello, se llevó a cabo un diseño factorial 2 (niveles factor 1) x 3 (niveles factor 2) x 3 (número de observaciones), se realizó un análisis de varianza
ANOVA y se identificaron diferencias significativas mediante la prueba de Tukey, con un nivel de significancia $\mathrm{P}<0,05$. Para evaluar el ajuste de los modelos matemáticos con los datos experimentales, se utilizaron los coeficientes estadísticos, suma de los errores al cuadrado (SSE) y coeficiente de determinación $\mathrm{R}^{2}$. Los mejores ajustes fueron aquellos modelos que presentaron los valores más bajos de SSE y valores de $\mathrm{R}^{2}$ más cercanos a 1. Los modelos fueron resueltos mediante Excel (2013), con la función SOLVER. Con el fin de evaluar el efecto de la temperatura y la incorporación de PZD en las difusividades efectivas y el tiempo de secado en el período de velocidad decreciente calculadas para espagueti funcional, se realizó una correlación de Pearson, con un nivel de significancia $\alpha=0,05$. Se empleó el software SPSS.18. 


\section{RESULTADOS Y DISCUSIÓN}

Caracterización de la materia prima. PZD presentó un contenido de humedad, proteína, fibra dietaria, grasa y cenizas de 13,08 $\pm 0,07 \mathrm{~g} / 100 \mathrm{~g} ; 15,08 \pm 0,06 \mathrm{~g} / 100 \mathrm{~g} ; 34,12 \pm 0,05 \mathrm{~g} / 100 \mathrm{~g} ; 1,58$ $\pm 0,01 \mathrm{~g} / 100 \mathrm{~g}$ y $9,37 \pm 0,12 \mathrm{~g} / 100 \mathrm{~g}$, respectivamente, mayor a lo reportado por Escalada Pla et al. (2007) y Noor \& Komathi (2009); sin embargo, el contenido de fibra dietaria obtenida en este estudio fue menor. Las diferencias observadas entre los resultados de este estudio y el de otros autores, se deben a múltiples factores, como las condiciones agroclimáticas del cultivo, el estado de madurez del fruto, así como la variedad.

Al comparar la composición proximal de PZD con la obtenida para la sémola de trigo (contenido de humedad $=11,14 \pm 0,03 \mathrm{~g} / 100 \mathrm{~g}$, grasa $=1,23 \pm 0,05 \mathrm{~g} / 100 \mathrm{~g}$, proteína $=12,05 \pm 0,08 \mathrm{~g} / 100 \mathrm{~g}$, cenizas $=1,01 \pm 0,03 \mathrm{~g} / 100 \mathrm{~g}$ y fibra dietaria $=2,04 \pm 0,02 \mathrm{~g} / 100 \mathrm{~g}$ b.s.), se evidenció que PZD presentó mayor contenido en fibra dietaria total, proteína y cenizas. El mismo resultado ha sido reportado por Mirhosseini et al. (2015), para pastas enriquecidas por PZD.

Finalmente, respecto al contenido de carotenoides totales, para PZD, se obtuvo un valor de $511,16 \pm 2,97 \mu \mathrm{g} / \mathrm{g}$, mayor a lo reportado por Carvalho et al. (2012), 404,98 $\pm 17,37 \mu \mathrm{g} / \mathrm{g} \mathrm{y}$, para la sémola de trigo, un valor de $0,71 \pm 0,87 \mu \mathrm{g} / \mathrm{g}$, mayor a lo reportado por Kandlakunta et al. (2008), 0,46 $\pm 0,054 \mu \mathrm{g} / \mathrm{g}$. Esto muestra una ventaja de PZD respecto a la sémola de trigo, en cuanto al contenido de carotenoides totales. Este parámetro es un indicador del aporte nutricional en un micronutriente tan importante, como el $\beta$-caroteno, al ser precursor de vitamina $A$, lo cual, también se constituye en un indicador de calidad. Es por esto, PZD podría aportar propiedades nutracéuticas, como mejorar la respuesta inmune y la reducción del riesgo de enfermedades cardiovasculares y otras, como el cáncer, la aterosclerosis y las cataratas (González et al. 2001).

Por lo tanto, PZD es una fuente disponible de sustancias biológicamente aprovechables que, al ser incluida en la formulación de alimentos a base de harina de trigo, mejora las características nutricionales.
Modelamiento matemático de las curvas de secado y determinación del coeficiente de difusividad. Seis modelos matemáticos más usados en la literatura fueron utilizados para representar las cinéticas de secado experimentales de espagueti enriquecido con pulpa de zapallo deshidratada, en el período de velocidad decreciente. Los parámetros que se tuvieron en cuenta para el modelamiento matemático, se muestran en la tabla 2, obtenidos experimentalmente. En la tabla 3, se muestran las constantes obtenidas para cada uno, junto con los valores de SSE y $\mathrm{R}^{2}$ respectivos. Para todos los casos, se evidenció que el modelo con mayor ajuste fue el Logarítmico; sin embargo, para espagueti PZD-5 secado a $60^{\circ} \mathrm{C}$, el modelo que presentó mayor $\mathrm{R}^{2}$ fue el de Henderson \& Pabis. En consecuencia, estos fueron usados para modelar el período de secado, a velocidad decreciente. En la figura 1 , se muestran las curvas experimentales y las predichas de los espaguetis secos, a dos temperaturas diferentes $\left(50\right.$ y $\left.60^{\circ} \mathrm{C}\right)$.

La difusividad efectiva $\left(D_{e}\right)$ representa la facilidad con que el agua se difunde en el medio que lo rodea y depende de la naturaleza del objeto que la contiene y del medio circundante; sin embargo, esta se ve afectada principalmente por la temperatura, además de la presión.

De aumentó desde $1,19 \times 10^{-7} \mathrm{~cm}^{2} \mathrm{~s}^{-1}$ hasta $3,27 \times 10^{-7} \mathrm{~cm}^{2} \mathrm{~s}^{-1}$, al aumentar PZD, desde 5 a $10 \%$ y aumentó por efecto de la temperatura, desde $1,88 \times 10-7 \mathrm{~cm}^{2} \mathrm{~s}^{-1}\left(50^{\circ} \mathrm{C}\right)$ hasta $2,82 \times 10^{-7} \mathrm{~cm}^{2} \mathrm{~s}^{-1}$ $\left(60^{\circ} \mathrm{C}\right)$, lo cual, se corroboró con los resultados de la correlación de Pearson realizada. PZD mostró una relación lineal con $\mathrm{De}$, con un valor de $r=0,763$ y una significancia de 0,004. Asimismo, la temperatura tuvo una relación lineal con $\mathrm{D}_{\mathrm{e}}$, con un valor $\mathrm{r}=0,630$ y una significancia de 0,028 . Es por esto, que los tiempos de secado para alcanzar la humedad final de $0,13 \mathrm{~g} / \mathrm{g}$ disminuyeron con el aumento de la temperatura y con el aumento de PZD (PZD5-50: 5,00h; PZD5-60: 3,40h; PZD10-50: 4,10h y PZD10-60: 2,80h). Los rangos de tiempo obtenidos en el presente estudio fueron similares a los obtenidos por Larrosa et al. (2016), para espagueti libre de gluten, secado a $50^{\circ} \mathrm{C}(3,20 \mathrm{~h})$.

Tabla 2. Parámetros considerados para el modelamiento matemático del secado de espagueti funcional con sustitución parcial de sémola de trigo T. durum por pulpa de zapallo deshidratada.

\begin{tabular}{|l|c|c|c|c|c|}
\hline \multirow{2}{*}{ Exp. } & \multicolumn{5}{|c|}{ Parámetro } \\
\cline { 2 - 6 } & $\mathbf{C i}(\mathbf{g})$ & $\mathbf{D}(\mathbf{m m})$ & $\mathbf{M}_{\mathbf{0}} \mathbf{( g / g )}$ & $\left.\mathbf{M}_{\mathbf{e}} \mathbf{( g / g}\right)$ & $\mathbf{t}_{\mathbf{c}}(\mathbf{h})$ \\
\hline PZD-5 $\left(50^{\circ} \mathrm{C}\right)$ & $300,03 \pm 1,01$ & $2,00 \pm 0,50$ & $0,32 \pm 0,01$ & $0,080 \pm 0,03$ & $2,00 \pm 0,00$ \\
\hline PZD-5 $\left(60^{\circ} \mathrm{C}\right)$ & $300,01 \pm 1,03$ & $2,00 \pm 0,50$ & $0,28 \pm 0,02$ & $0,075 \pm 0,01$ & $1,00 \pm 0,03$ \\
\hline PZD-10 $\left(50^{\circ} \mathrm{C}\right)$ & $300,01 \pm 0,97$ & $2,00 \pm 0,50$ & $0,35 \pm 0,05$ & $0,087 \pm 0,01$ & $1,00 \pm 0,00$ \\
\hline PZD-10 $\left(60^{\circ} \mathrm{C}\right)$ & $300,05 \pm 0,92$ & $2,00 \pm 0,50$ & $0,27 \pm 0,03$ & $0,075 \pm 0,02$ & $1,00 \pm 0,02$ \\
\hline
\end{tabular}

Exp: Experimento, PZD: Pulpa de Zapallo Deshidratada, Ci: Cargar inicial, D: Diámetro, M0: Contenido de humedad inicial, Me: Contenido de humedad de equilibrio y tc: tiempo de secado en el período de velocidad constante. Los datos corresponden al promedio \pm $\mathrm{DE}(\mathrm{n}=3)$. 
Tabla 3. Resultados estadísticos obtenidos de los diferentes modelos de secado para el período de velocidad decreciente.

\begin{tabular}{|c|c|c|c|c|c|c|c|c|}
\hline \multirow{2}{*}{ Exp. } & \multirow{2}{*}{$\begin{array}{c}\text { No. } \\
\text { Modelo }\end{array}$} & \multicolumn{5}{|c|}{ Parámetros del modelo } & \multirow{2}{*}{$\mathbf{R}^{2}$} & \multirow{2}{*}{ SSE } \\
\hline & & $\mathbf{k}$ & $\mathbf{k}_{1}$ & $\mathrm{n}$ & a & b & & \\
\hline \multirow{6}{*}{$\begin{array}{l}\text { PZD-5 } \\
\left(50^{\circ} \mathrm{C}\right)\end{array}$} & 01 & 0,33 & & & & & 0,516 & 0,0054 \\
\hline & 02 & 0,60 & & 0,59 & & & & 0,0004 \\
\hline & 03 & 0,19 & & & 0,57 & & 0,996 & 0,0006 \\
\hline & 04 & 0,73 & & & 1,38 & 0,17 & 0,999 & 0,0001 \\
\hline & 05 & 0,62 & 0,00 & & 1,03 & 0,16 & 0,986 & 0,0002 \\
\hline & 06 & 0,93 & & & 0,28 & & 0,803 & 0,0022 \\
\hline \multirow{6}{*}{$\begin{array}{l}\text { PZD-5 } \\
\left(60^{\circ} \mathrm{C}\right)\end{array}$} & 01 & 0,48 & & & & & 0,769 & 0,0018 \\
\hline & 02 & 0,63 & & 0,75 & & & 0,939 & 0,0012 \\
\hline & 03 & 0,33 & & & 0,65 & & 0,994 & 0,0010 \\
\hline & 04 & 0,33 & & & 0,65 & 0,00 & 0,987 & 0,0010 \\
\hline & 05 & 0,34 & 0,33 & & 0,10 & 0,55 & 0,975 & 0,0010 \\
\hline & 06 & 1,34 & & & & 0,28 & 0,985 & 0,0020 \\
\hline \multirow{6}{*}{$\begin{array}{l}\text { PZD-10 } \\
\left(50^{\circ} \mathrm{C}\right)\end{array}$} & 01 & 0,38 & & & & & 0,959 & 0,0008 \\
\hline & 02 & 0,48 & 0,82 & & & & 1,000 & 0,0000 \\
\hline & 03 & 0,31 & & & 0,77 & & 1,000 & 0,0000 \\
\hline & 04 & 0,32 & & & 0,77 & 0,01 & 1,000 & 0,0000 \\
\hline & 05 & 0,48 & 0,28 & & 0,22 & 0,59 & 1,000 & 0,0000 \\
\hline & 06 & 0,38 & & & 0,97 & & 0,959 & 0,0008 \\
\hline \multirow{6}{*}{$\begin{array}{l}\text { PZD-10 } \\
\left(60^{\circ} \mathrm{C}\right)\end{array}$} & 01 & 0,45 & & & & & 0,966 & 0,0019 \\
\hline & 02 & 0,53 & 0,85 & & & & 0,993 & 0,0004 \\
\hline & 03 & 0,37 & & & 0,81 & & 0,992 & 0,0004 \\
\hline & 04 & 0,40 & & & 0,82 & 0,01 & 0,993 & 0,0004 \\
\hline & 05 & 0,36 & 1,10 & & 0,74 & 0,19 & 0,993 & 0,0004 \\
\hline & 06 & 1,03 & & & 0,33 & & 0,992 & 0,0004 \\
\hline
\end{tabular}

Exp: Experimento; PZD: Pulpa de Zapallo Deshidratada; k: constante de secado; a, b, c y n: coeficientes de ajuste, SSE: Suma de Errores al Cuadrado, $\mathrm{R}^{2}$ : coeficiente de determinación. 

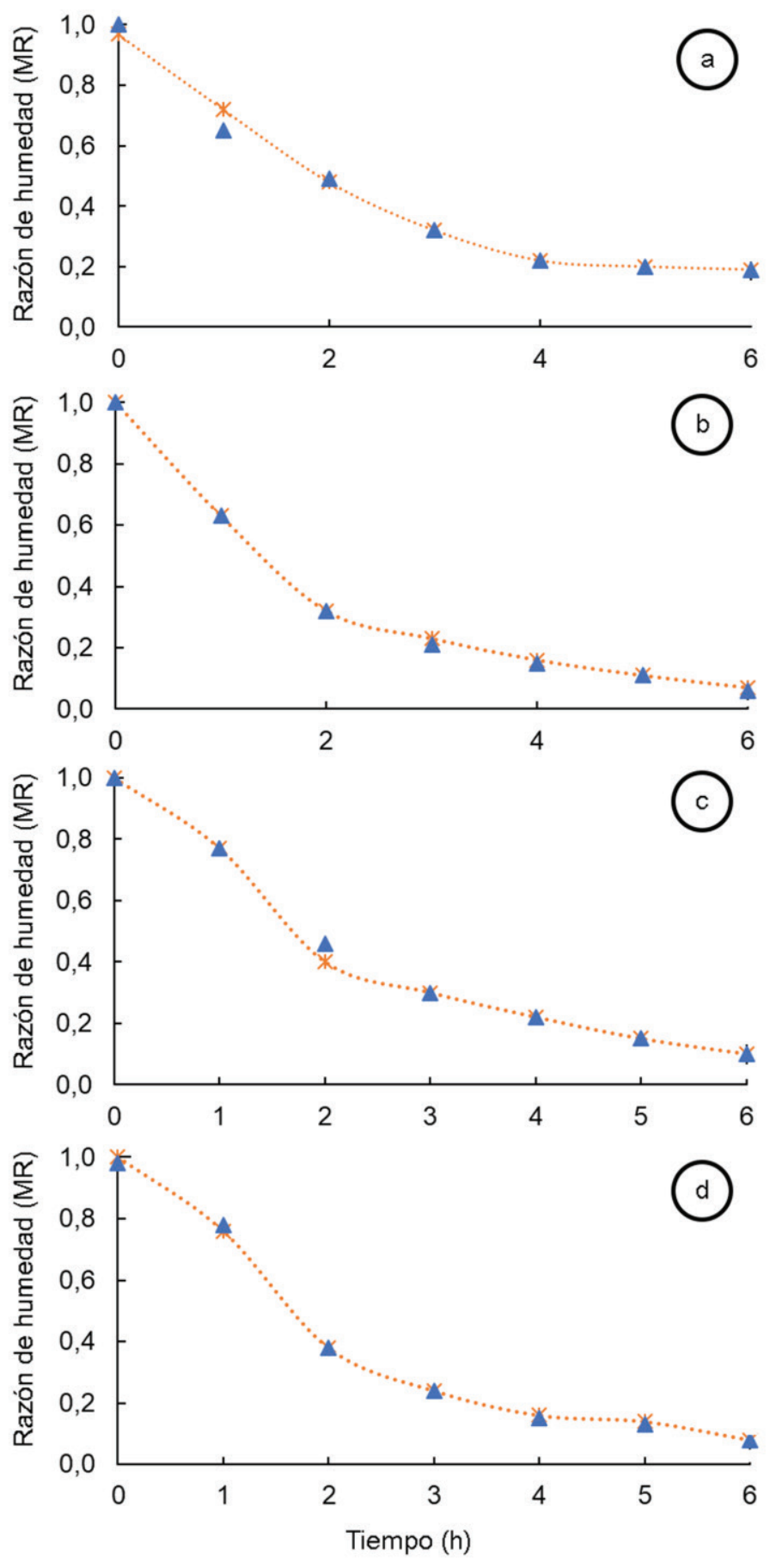

$\Delta$ Exp …*...Pred

Figura 1. Comparación entre la relación de humedad predicha y experimental durante el secado de espagueti enriquecido con PZD, a dos temperaturas $\left(\boldsymbol{\Delta}\right.$ Datos experimentales, ${ }^{*}$ Datos predichos por el modelo. a. PZD-5 a $50^{\circ} \mathrm{C}$ (Logarítmico); b. PZD-5 a $60^{\circ} \mathrm{C}($ Henderson \& Pabis); c. PZD-10 a $50^{\circ} \mathrm{C}$ (Logarítmico); d. PZD-10 a $60^{\circ} \mathrm{C}$ (Logarítmico). 
La incorporación de PZD a la formulación de espagueti pudo haber disminuido la resistencia de transferencia de masa interna del producto, es decir, que PZD pudo haber aumentado la porosidad, lo que incrementó la difusividad del agua (Waananen, 1996). En la literatura, se reportan valores de $4,30 \times 10^{-7} \mathrm{~cm}^{2} \mathrm{~s}^{-1}$, para espagueti con $100 \%$ sémola de trigo, secado a $40^{\circ} \mathrm{C}$ (Mercier et al. 2013), mayores a los obtenidos para PZD de este estudio. Entre otras razones, dicho resultado, se puede deber a que los autores emplearon humedades relativas menores $(65 \%)$ a las utilizadas para esta investigación $(70 \%)$.

Caracterización de la calidad del espagueti. El análisis de varianza realizado para evaluar el efecto de PZD y de la temperatura de secado sobre algunas variables que definen la calidad del espagueti seco, se muestran en la tabla 4. Esta demostró, que existe una influencia altamente significativa de los factores de forma independiente sobre el contenido de humedad, tiempo de cocción, sólidos perdidos en el agua de cocción y ganancia de peso. Para la variable contenido de carotenoides totales, solo el factor PZD presentó un efecto significativo $(\mathrm{P}<0,05)$. Respecto al efecto de la interacción de los factores fue significativo para las variables tiempo de cocción y sólidos perdidos en el agua de cocción $(\mathrm{P}<0,05)$. Asimismo, también se observa que el efecto de PZD fue positivo para las variables de respuesta contenido de humedad $(0,506)$, sólidos perdidos en el agua de cocción (efecto $=0,11$ ) y contenido de carotenoides totales (efecto $=82,89$ ). Para las demás variables el efecto fue negativo.

Tabla 4. Análisis de varianza para cada variable dependiente.

\begin{tabular}{|l|c|c|c|c|c|c|}
\hline \multirow{2}{*}{ VR } & \multicolumn{2}{|c|}{ PZD } & \multicolumn{2}{c|}{ T } & \multicolumn{2}{c|}{ PZD*T } \\
\cline { 2 - 7 } & F-valor & P-valor & F-valor & P-valor & F-valor & P-valor \\
\hline CH & 5,75 & 0,031 & 199,79 & 0,000 & 0,32 & 0,581 \\
\hline TC & 13190,93 & 0,000 & 1450,42 & 0,000 & 7,59 & 0,015 \\
\hline SPC & 27,62 & 0,000 & 73,65 & 0,000 & 27,62 & 0,000 \\
\hline GP & 183,76 & 0,000 & 6738,65 & 0,000 & 0,00 & 1,000 \\
\hline CCT & 156,82 & 0,000 & 0,26 & 0,615 & 0,64 & 0,439 \\
\hline
\end{tabular}

VR: Variable de Respuesta, PZD: Pulpa de Zapallo Deshidratada (g/100g harina), T: Temperatura; CH: Contenido de Humedad, TC: Tiempo de Cocción, SP: Sólidos Perdidos en el agua de Cocción, GP: Ganancia de Peso, CCT: Contenido de Carotenoides Totales. El efecto es significativo en un nivel $\mathrm{P}<0,05$.

Tabla 5. Caracterización de la calidad del espagueti enriquecido con pulpa de zapallo deshidratada, secado a diferentes temperaturas.

\begin{tabular}{|c|c|c|c|c|c|}
\hline Exp. & $\begin{array}{c}\mathrm{CH} \\
(\mathrm{g} / 100 \mathrm{~g}) \\
\end{array}$ & $\begin{array}{c}\text { TC } \\
(\mathrm{min})\end{array}$ & SPC (g/100g) & $\begin{array}{c}\text { GP } \\
(\mathrm{g} / 100 \mathrm{~g})\end{array}$ & $\begin{array}{c}\text { CCT } \\
(\mathrm{g} / 100 \mathrm{~g}) \\
\end{array}$ \\
\hline $\begin{array}{c}\text { PZD-0 } \\
\left(50^{\circ} \mathrm{C}\right)\end{array}$ & $10,35 \pm 0,39^{\mathrm{aA}}$ & $8,32 \pm 0,10^{\mathrm{aA}}$ & $0,88 \pm 0,00^{\mathrm{bA}}$ & $137,00 \pm 0,65^{\mathrm{aA}}$ & $22,39 \pm 3,63^{\mathrm{cA}}$ \\
\hline $\begin{array}{c}\text { PZD-0 } \\
\left(60^{\circ} \mathrm{C}\right)\end{array}$ & $8,23 \pm 0,01^{\mathrm{aB}}$ & $7,11 \pm 0,0^{\mathrm{aB}}$ & $0,65 \pm 0,0^{\mathrm{bB}}$ & $107,00 \pm 0,75^{\mathrm{aB}}$ & $23,33 \pm 3,63^{\mathrm{cA}}$ \\
\hline $\begin{array}{c}\text { PZD-5 } \\
\left(50^{\circ} \mathrm{C}\right)\end{array}$ & $11,14 \pm 0,07^{\mathrm{aA}}$ & $6,20 \pm 0,0^{\mathrm{bA}}$ & $0,96 \pm 0,0^{\mathrm{aA}}$ & $133,00 \pm 0,65^{\mathrm{bA}}$ & $44,42 \pm 5,29^{\mathrm{bA}}$ \\
\hline $\begin{array}{c}\text { PZD-5 } \\
\left(60^{\circ} \mathrm{C}\right)\end{array}$ & $8,38 \pm 0,00^{\mathrm{aB}}$ & $5,14 \pm 0,00^{\mathrm{bB}}$ & $0,76 \pm 0,00^{\mathrm{aB}}$ & $104,00 \pm 0,75^{\mathrm{bB}}$ & $44,75 \pm 4,30^{\mathrm{bA}}$ \\
\hline $\begin{array}{c}\text { PZD-10 } \\
\left(50^{\circ} \mathrm{C}\right)\end{array}$ & $10,97 \pm 0,72^{\mathrm{aA}}$ & $4,19 \pm 0,,^{04 \mathrm{c} A}$ & $0,88 \pm 0,0^{\mathrm{aA}}$ & $131,00 \pm 0,71^{\mathrm{cA}}$ & $110,56 \pm 5,98^{\mathrm{aA}}$ \\
\hline $\begin{array}{c}\text { PZD-10 } \\
\left(60^{\circ} \mathrm{C}\right)\end{array}$ & $8,61 \pm 0,1^{\mathrm{aB}}$ & $3,18 \pm 0,00^{\mathrm{cB}}$ & $0,87 \pm 0,00^{\mathrm{aB}}$ & $101,00 \pm 0,80^{\mathrm{cB}}$ & $100,95 \pm 4,75^{\mathrm{aA}}$ \\
\hline
\end{tabular}

Exp: Experimento; PZD: Pulpa de Zapallo Deshidratada, CH: Contenido de Humedad, TC: Tiempo de Cocción, SP: Sólidos Perdidos en el agua de Cocción, GP: Ganancia de Peso, CCT: Contenido de Carotenoides Totales. Los valores corresponden al promedio $\pm \operatorname{DE}(n=3)$. Diferentes letras $(a, b, c \ldots)$ en la misma columna indican diferencias significativas por efecto de PZD en la formulación $(\mathrm{P}<0,05)$ y diferentes letras $(\mathrm{A}, \mathrm{B}, \mathrm{C} \ldots)$ en la misma columna indican diferencias significativas por efecto de la temperatura $(\mathrm{P}<0,05)$. 
En la tabla 5, se puede observar cómo la muestra control tiene los valores más altos de tiempo de cocción y ganancia de peso. A medida que aumenta PZD en la formulación el tiempo de cocción disminuye, mientras que aumentan significativamente los sólidos perdidos en el agua de cocción $(\mathrm{P}<0,05)$. Este comportamiento, se puede deber a la disrupción física de la matriz del gluten por la fibra, proporcionando un camino más fácil para la absorción de agua y, en consecuencia, un menor tiempo de cocción (Manthey \& Schorno, 2002).

Por otro lado, la disminución de las propiedades de cocción con el aumento de la temperatura, se debe al aumento en la resistencia de la red formada entre el gluten y el almidón. Con anterioridad, el mismo comportamiento ya ha sido reportado. Dexter \& Morgan (1981) evidenciaron en su estudio, que las pérdidas por cocción se ven afectadas por las condiciones de secado, siendo estas más bajas cuando se secan a altas temperaturas; sin embargo, encontraron que dicho comportamiento no se ve afectado por las propiedades de gelatinización del almidón, ya que no cambia con la condición de secado. Posteriormente, Padalino et al. (2016) obtienen que la calidad de cocción del espagueti mejora con el aumento de la temperatura de secado, lo cual, lo relacionaron con el aumento de la densidad, tanto de la reticulación física como la reticulación química de la matriz proteica. A diferencia de lo anterior, Samritthisuth \& Rattanasumawong (2013) no reportaron diferencias significativas en el tiempo de cocción, por efecto de la temperatura de secado. Finalmente, respecto al contenido de carotenoides totales, su incremento con la adición de PZD en la formulación y el aumento de la temperatura de secado, se debe al aumento de la solubilidad del betacaroteno. Por otro lado, al efectuarse el secado a menos temperatura, aumenta el tiempo de exposición de la muestra al oxígeno, lo que ocasiona que sea menor el contenido de betacaroteno, a menor temperatura. El mismo comportamiento fue reportado por Ihns et al. (2011), en albaricoque seco, a diferentes temperaturas.

Con este estudio, se obtuvieron modelos matemáticos que representan las cinéticas de secado de espagueti enriquecido con pulpa de zapallo deshidratada (5 y $10 \mathrm{~g} / 100 \mathrm{~g}$ harina), con un grado de ajuste $\mathrm{R}^{2}>0,9$, los cuales, corresponden al modelo Logarítmico, en su mayoría. También, se evidenció que tanto PZD como la temperatura afectaron el tiempo de secado, que disminuyó con el aumento de ambos factores. PZD presentó mayor correlación positiva con De, respecto de la temperatura de secado. Mediante los modelos obtenidos, se logra obtener una ecuación que predice el contenido de humedad, en determinado tiempo. Dado a la especificidad del modelo y a las características propias del material, como porosidad, conductividad térmica, así como a las condiciones a las que fue realizada, para obtener un modelo más general, que incluya dichos factores, se deben emplear otros modelos físicos, que permitan su evaluación; de esta manera, se aumenta el rango de aplicabilidad del modelo obtenido.

Finalmente, se logró evaluar el efecto de la sustitución parcial de la sémola de trigo por pulpa de zapallo deshidratada y de la temperatura de secado sobre algunas propiedades, que definen la calidad del espagueti. Se obtuvo una disminución de la calidad de cocción y aumento del contenido de carotenoides totales con el aumento de PZD y una disminución de los sólidos perdidos en el agua de cocción y aumento del contenido de carotenoides con el aumento de la temperatura.

Conflictos de intereses: El manuscrito fue desarrollado y revisado con la participación de todos los autores, quienes declaramos que no existe conflicto de intereses que ponga en riesgo la validez de los resultados presentados. Financiación: Este estudio fue financiado por la Facultad de Ingeniería y Administración de la Universidad Nacional de Colombia Sede Palmira mediante el proyecto Hermes 35763.

\section{REFERENCIAS}

1. AMERICAN ASSOCIATION OF CEREAL CHEMISTS, AACCI. 2000. 66-50.01. 11th Ed, Pasta and Noodle Cooking Quality-Firmness. St. Paul, MN, USA

2. ASSOCIATION OF OFFICIAL ANALYTICAL CHEMISTS, AOAC. 2003. Official Methods of Analysis, Washington D.C., USA

3. AGRAWAL, Y.C.; SINGH, R.D. 1977. Thin layer drying studies for short grain rice. Transactions of the American society of Agricultural and Biological Engineers (USA). 77(3531):1-13.

4. ALJAHANI, A.; CHEIKHOUSMAN, R. 2017. Nutritional and sensory evaluation of pumpkin-based (Cucurbita maxima) functional juice. Nutrition and Food Science (United Kingdom). 47(3):346-356. https://doi.org/10.1108/NFS07-2016-0109

5. ASTAÍZA, M.; RUÍZ, L.; ELIZALDE, A. 2010. Elaboración de pastas alimenticias enriquecidas a partir de harina de quinua (Chenopodium quinoa wild.) y zanahoria (Daucus carota). Biotecnología en el Sector Agropecuario y Agroindustrial. (Colombia). 8(1):43-53

6. BERGANTIN, C.; MAIETTTI, A.; TEDESCHI, P.; FONT, G.; MANYES, L.; MARCHETTI, N. 2018. HPLC-UV/VisAPCI-MS/MS determination of major carotenoids and their bioaccessibility from "Delica" (Cucurbita maxima) and "Violina" (Cucurbita moschata) pumpkins as food traceability markers. Molecules. (Switzerland). 23:1-13. https://doi. org/10.3390/molecules23112791

7. CHHINNAN, M.S. 1984. Evaluation of selected mathematical models for describing thin-layer drying of in-shell pecans. Transactions Am. Soc. Agricultural and Biological Engineers. (USA). 27(2):610-615. https://doi. org $/ 10.13031 / 2013.32837$ 
8. CRANK, J. 1975. The Diffusion Equations. Oxford University. The mathematics of Diffusión. Great Britain, England. $2^{\text {a }}$ Ed., p.5-10.

9. DE CARVALHO, L.M.J.; GOMES, P.B.; GODOY, R.L. DE O.; PACHECO, S.; DO MONTE, P.H.F.; DE CARVALHO, J.L.V.; NUT'TI, M.R.; LIMA NEVES, A.C.; RODRIGUES ALVES VIEIRA, A.C.; RAMOS, S.R.R. 2012. Total carotenoid content, $\alpha$-carotene and $\beta$-carotene, of landrace pumpkins (Cucurbita moschata Duch): A preliminary study. Food Research International. (Netherlands). 47(2):337-340. https//doi.org/10.1016/j.foodres.2011.07

10. DE PILLI, T.; GIULIANI, R.; DEROSSI, A.; SEVERINI, C. 2009. Study of cooking quality of spaghetti dried through microwaves and comparison with hot air dried pasta. J. Food Engineering. (USA). 95(3):453-459. https//:doi. org/10.1016/j.jfoodeng.2009.06.005

11. DE TEMMERMAN, J.; VERBOVEN, P.; NICOLAI, B.; RAMON, H. 2007. Modelling of transient moisture concentration of semolina pasta during air drying. J. Food Engineering. 80(3):892-903. https://doi.org/10.1016/j. jfoodeng.2006.08.004

12. DEXTER, J.E.; MATSUO, R.R.; MORGAN, B.C. 1981. High Temperature Drying: Effect on Spaghetti Properties. J. Food Science. (USA). 46:1741-1746. https://doi. org/10.1111/j.1365-2621.1981.tb04476.x

13. ERTEKIN, C.; YALDIZ, O. 2004. Draying of eggplant and selection of a suitable thin layer drying model, J. Food Engineering. 63(4):349-359. https://doi.org/10.1016/ j.jfoodeng.2003.08.007

14. ESCALADA PLA, M.F.; PONCE, N.M.; STORTZ, C.A.; GERSCHENSON, L.N.; ROJAS, A.M. 2007. Composition and functional properties of enriched fiber products obtained from pumpkin (Cucurbita moschata Duchesne ex Poiret). LWT- Food Science and Technology. (Switzerland). 40:1176-1185. https://doi.org/10.1016/j.lwt.2006.08.006

15. GEANKOPLIS, C.J. 1998. Procesos de transporte y operaciones unitarias. Compañía Editorial Continental, S.A. de C.V.

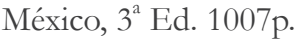

16. GONZÁLEZ, E.; MONTENEGRO, M.A.; NAZARENO, M.A. 2001. Carotenoid composition and vitamin A value of an Argentinian squash (Cucurbita moschata). Arch. Latinoam. Nutrición (Venezuela), 51(4):395-399.

17. HENDERSON, S.M. 1974. Progress in developing the thinlayer drying equation. Transactions Am. Soc. Agricultural and Biological Engineers. 17(6):1167-1168/1172 https:/ / doi.org/10.13031/2013.37052
18. IHNS, R.; DIAMANTE, L.M.; SAVAGE, G P.; VANHANEN, L. 2011. Effect of temperature on the drying characteristics, colour, antioxidant and beta-carotene contents of two apricot varieties. Internal J. Food Science \& Technology (United Kingdom). 46(2):275-283. https://doi. org/10.1111/j.1365-2621.2010.02506.x

19. KANDLAKUNTA, B.; RAJENDRAN, A.; THINGNGANING, L. 2008. Carotene content of some common (cereals, pulses, vegetables, spices and condiments) and unconventional sources of plant origin. Food Chemistry. (Netherlands), 106(1):85-89. https://doi. org/10.1016/j.foodchem.2007.05.071

20. LARROSA, V.; LORENZO, G.; ZARITZKY, N.; CALIFANO, A. 2016. Modelado matemático del secado de pastas libres de gluten en relación a la temperatura y humedad relativa del aire. Rev. Laboratorio Tecnológico del Uruguay. 11:5458

21. LU, A.; YU, M.; FANG, Z.; XIAO, B.; GUO, L.; WANG, W.; LI, J.; WANG, S.; Zhang, Y. 2019. Preparation of the controlled acid hydrolysates from pumpkin polysaccharides and their antioxidant and antidiabetic evaluation. International J. Biological Macromolecules. (Netherlands). 121:261-269. https://doi.org/10.1016/j.ijbiomac.2018.09.158

22. MANTHEY, F.A.; SCHORNO, A. 2002. Physical and cooking quality of spaghetti made from whole-wheat durum. Cereal Chemestry. (United States). 79:504-510. https:// doi.org/10.1094/CCHEM.2002.79.4.504

23. MERCIER, S.; MORESOLI, C.; VILLENEUVE, S.; MONDOR, M.; MARCOS, B. 2013. Sensitivity analysis of parameters affecting the drying behaviour of durum wheat pasta. J. Food Engineering. 118(1):108-116. https:/ / doi.org/10.1016/j.jfoodeng.2013.03.024

24. MINAROVIČOVÁ, L.; LAUKOVÁ, M.; KOHAJDOVÁ, Z.; KAROVIČOVÁ, J.; KUCHTOVÁ, V. 2017. Effect of pumpkin powder incorporation on cooking and sensory parameters of pasta. Potravinarstvo Slovak J. Food Sciences. 11(1):373-379. https://doi.org/10.5219/743

25. MIRHOSSEINI, H.; ABDUL RASHID, N.F.; TABATABAEE AMID, B.; CHEONG, K.W.; KAZEMI, M.; ZULKURNAIN, M. 2015. Effect of partial replacement of corn flour with durian seed flour and pumpkin flour on cooking yield, texture properties, and sensory attributes of gluten free pasta. LWT - Food Science and Technology. 63(1):184-190. https://doi.org/10.1016/j.lwt.2015.03.078

26. NOOR, A.A.; KOMATHI, C.A. 2009. Physicochemical and Functional Properties of Peeled and Unpeeled Pumpkin Flour. J. Food Science. 74(7):328-333. https://doi. org/10.1111/j.1750-3841.2009.01298.x 
27. ORDOÑEZ - SANTOS, L.E.; HURTADO, P.; RÍOS, O.D.; ARIAS, M.E. 2014. Concentración de carotenoides totales en residuos de frutas tropicales, Producción+Limpia (Colombia). 9(1):91-98

28. ORREGO, C.E.; VALLEJO, D.; MANRIQUE, D.L.; GONZÁLEZ, J.D.; OCAMPO, J.C. 2016. Inactivación de peroxidasa en banano (Musa paradisiaca) por medio de tratamiento térmico y ultrasónico. Agronomía Colombiana. 34(1Supl.):S457-S460

29. OZDEMIR, M.; DEVRES, Y.O. 1999. The thin layer drying characteristics of hazelnuts during roasting. J. Food Engineering. 42(4):225-233. https://doi.org/10.1016/ S0260-8774(99)00126-0

30. PADALINO, L.; CALIANDRO, R.; CHITA, G.; CONTE, A.; DEL NOBILE, M. A. 2016. Study of drying process on starch structural properties and their effect on semolina pasta sensory quality. Carbohydrate Polymers. (Inglaterra). 153:229-235. https://doi.org/10.1016/ j.carbpol.2016.07.102

31. POTOSÍ-CALVACHE, D.C.; VANEGAS-MAHECHA, P.; MARTINEZ, H.A. 2017. Convective drying of squash (Cucurbita moschata): Influence of temperature and air velocity on effective moisture diffusivity, carotenoid content and total phenols. DYNA (Colombia). 84(202):112119.https://doi.org/10.15446/dyna.v84n202.63904
32. SAMRITTHISUTH, C.; RATTANASUMAWONG, S. 2013. Effect of drying temperature on rehydration properties of dried rice noodle. Conference peper, Procedings of the $51^{\text {st }}$ Kasetsart University Annual Conference, Bangkok, Thailand, 5 -7 February 2013, p.175 ref 8

33. SHARAF-ELDEEN, O.; BLAISDELL, Y.I.; SPAGNA, G. 1980. A model for ear corn drying. Transactions Am. Soc. Agricultural and Biological Engineers. 23(5):1261-1271. https://doi.org/10.13031/2013.34757

34. VEGA, A.; FITO, P. 2005. Modelado de la cinética de secado del pimiento Rojo (Capsicum annuиm L. cv Lamuyo). Inform. Tecnológica (Chile). 16(6):3-11. https://doi.org/10.4067/ S0718-07642005000600002

35. WAANANEN, K.M. 1996. Effect of porosity on moisture diffusion during drying of pasta. J. Food Engineering. 28(2):121-137. https://doi.org/10.1016/02608774(94)00082-4

36. WESTERMAN, P.W.; WHITE, G.M.; ROSS, I.J. 1973. Relative humidity effect on the high temperature drying of shelled corn. Transactions Am. Soc. Agricultural and Biological Engineers. 16:1136-1139. https://doi. org/10.13031/2013.37715

37. YAGCIOGLU, A.; DEGIRMENCIOGLU, A.; CAGATAY, F. 1999. Drying characteristics of laurel leaves under different drying conditions. In Proceedings of the 7th international congress on agricultural mechanization and energy, Adana, Turkey 